\title{
Constraints on Changes in Fundamental Constants from a Cosmologically Distant OH Absorber or Emitter
}

\author{
N. Kanekar, ${ }^{1, *}$ C. L. Carilli, ${ }^{1}$ G. I. Langston, ${ }^{1}$ G. Rocha,${ }^{2}$ F. Combes,${ }^{3}$ R. Subrahmanyan, ${ }^{4}$ J. T. Stocke, ${ }^{5}$ K. M. Menten, ${ }^{6}$ \\ F. H. Briggs, ${ }^{4,7}$ and T. Wiklind ${ }^{8}$ \\ ${ }^{1}$ National Radio Astronomy Observatory, Socorro, New Mexico 87801, USA \\ ${ }^{2}$ Cavendish Laboratory, Cambridge, CB3 OHE, United Kingdom \\ ${ }^{3}$ Observatoire de Paris, F-75014, Paris, France \\ ${ }^{4}$ Australia Telescope National Facility, NSW 1710, Australia \\ ${ }^{5}$ University of Colorado, Boulder, Colorado 80309, USA \\ ${ }^{6}$ Max-Planck-Institut für Radioastronomie, 53121 Bonn, Germany \\ ${ }^{7}$ Australian National University, ACT 2611, Australia \\ ${ }^{8}$ Space Telescope Science Institute, Baltimore, Maryland 21218, USA
}

(Received 3 June 2005; published 19 December 2005)

\begin{abstract}
We have detected the four $18 \mathrm{~cm} \mathrm{OH}$ lines from the $z \sim 0.765$ gravitational lens toward PMN J01340931. The 1612 and $1720 \mathrm{MHz}$ lines are in conjugate absorption and emission, providing a laboratory to test the evolution of fundamental constants over a large lookback time. We compare the $\mathrm{HI}$ and $\mathrm{OH}$ main line absorption redshifts of the different components in the $z \sim 0.765$ absorber and the $z \sim 0.685$ lens toward B0218 +357 to place stringent constraints on changes in $F \equiv g_{p}\left[\alpha^{2} / \mu\right]^{1.57}$. We obtain $[\Delta F / F]=\left(0.44 \pm 0.36^{\text {stat }} \pm 1.0^{\text {syst }}\right) \times 10^{-5}$, consistent with no evolution over the redshift range $0<$ $z \lesssim 0.7$. The measurements have a $2 \sigma$ sensitivity of $[\Delta \alpha / \alpha]<6.7 \times 10^{-6}$ or $[\Delta \mu / \mu]<1.4 \times 10^{-5}$ to fractional changes in $\alpha$ and $\mu$ over a period of $\sim 6.5 \mathrm{G} \mathrm{yr}$, half the age of the Universe. These are among the most sensitive constraints on changes in $\mu$.
\end{abstract}

DOI: 10.1103/PhysRevLett.95.261301

PACS numbers: 98.80.Es, 06.20.Jr, 33.20.Bx, 98.58.-w

Introduction. - A fairly generic feature of modern higher-dimensional theoretical models is that fundamental constants like the fine structure constant $\alpha$, the electronproton mass ratio $\mu \equiv m_{e} / m_{p}$, the proton gyromagnetic ratio $g_{p}$, etc. depend on the scale lengths of the extra dimensions of the theory. In the current theoretical framework, it is implausible that these scale lengths remain constant, implying that $\alpha, \mu$, etc. should vary with time. The detection of such changes provides an avenue to probe new and fundamental physics.

Null results have been obtained in all terrestrial studies of evolving constants, with atomic clocks and isotopic abundances in the Oklo natural fission reactor providing the tightest constraints on fractional changes in the fine structure constant $\left[(1 / \alpha)[\Delta \alpha / \Delta t]<4 \times 10^{-15}\right.$ per year, over three years [1], and $\Delta \alpha / \alpha<1.2 \times 10^{-7}$, over $\sim 1.8 \times 10^{9}$ years [2], respectively]. However, terrestrial measurements only probe fairly small fractions of the age of the Universe; astrophysical techniques are needed to examine the possibility of variations at earlier times [e.g., [3-7] ]. It is these techniques that provide tantalizing evidence for changes in $\alpha$; the "many-multiplet" method, applied to Keck telescope optical spectra, gives $\Delta \alpha / \alpha=$ $(-5.4 \pm 1.2) \times 10^{-6}$ over the redshift range $0.2<z<3.7$ [3]. However, a similar technique, applied to Very Large Telescope spectra, yields a conflicting result, $\Delta \alpha / \alpha=$ $(-0.6 \pm 0.6) \times 10^{-6}$, over $0.4<z<2.3$ [4]. Independent techniques are clearly needed as systematics appear to play a significant role in the current results.
The four $18 \mathrm{~cm}$ radio $\mathrm{OH}$ lines have very different dependences on $\alpha, \mu$, and $g_{p}$ and their redshifted frequencies can hence be compared to each other and to those of the $\mathrm{HI} 21 \mathrm{~cm}$ hyperfine line or $\mathrm{HCO}^{+}$rotational lines to measure any variation $[8,9]$. Even more interesting is the case of conjugate emission/absorption by the $18 \mathrm{~cm}$ satellite $\mathrm{OH}$ lines, detected in a single cosmologically distant object [at $z \sim 0.247$ toward PKS1413 + 135; [5,10] ]. Here, the pumping mechanism guarantees that the two lines arise from the same gas; a comparison can thus be made between the 1720 and $1612 \mathrm{MHz}$ redshifts without concerns about systematic motions between the clouds in which the different lines arise.

Only four redshifted $\mathrm{OH}$ main absorbers are currently known [11], with high resolution data only available for the $z \sim 0.685$ lens toward B0218 +357 [12]. Similarly, only one redshifted conjugate $\mathrm{OH}$ satellite system is known, at a relatively low redshift, $z \sim 0.247$; this corresponds to a lookback time of $\sim 2.9 \mathrm{Gyr}$, not much earlier than the time range probed by the Oklo reactor [13]. We report here the detection of all four $18 \mathrm{~cm} \mathrm{OH}$ lines in a new conjugate system at $z \sim 0.765$, corresponding to a lookback time of $\sim 6.7 \mathrm{G} \mathrm{yr}$.

Spectra and results. - The redshifted $\mathrm{OH} 18 \mathrm{~cm}$ lines from the $z \sim 0.765$ lens toward PMN J0134-0931 were observed simultaneously with the Green Bank Telescope (GBT) in October 2004 and January 2005, with an observing resolution of $\sim 1 \mathrm{~km} / \mathrm{s}$ (after Hanning smoothing). A separate GBT observation in January 2005 provided a high 
resolution spectrum in the redshifted $\mathrm{HI} 21 \mathrm{~cm}$ line, originally detected by [14]. The $\mathrm{HI} 21 \mathrm{~cm}$ and main line $\mathrm{OH}$ spectra (smoothed to resolutions of $\sim 5$ and $\sim 14.5 \mathrm{~km} / \mathrm{s}$, respectively, and resampled) are shown in Figs. 1(a) and 1(b), while the three panels of Fig. 2 show the 1720 and $1612 \mathrm{MHz}$ satellite spectra and the sum of 1720 and $1612 \mathrm{MHz}$ optical depths (all at a resolution of $\sim 4.7 \mathrm{~km} / \mathrm{s}$ ). PMN J0134-0931 is unresolved by the GBT beam; the above optical depths are the ratio of line flux density to continuum flux density for each transition (using the low optical depth limit). All spectra have a rootmean-square optical depth noise of $\sim 0.0018$, per $\sim 5 \mathrm{~km} / \mathrm{s}$ channel.

Besides the above, the redshifted $\mathrm{HCO}^{+} 2-1$ line was observed with the IRAM $30 \mathrm{~m}$ telescope and the Australia Telescope Compact Array (ATCA), the $6 \mathrm{~cm}$ ground state $\mathrm{H}_{2} \mathrm{CO}$ doublet lines with the GBT, and the $2 \mathrm{~cm}$ first rotationally excited state $\mathrm{H}_{2} \mathrm{CO}$ lines with the Very Large Array and the GBT. None of these transitions were de-
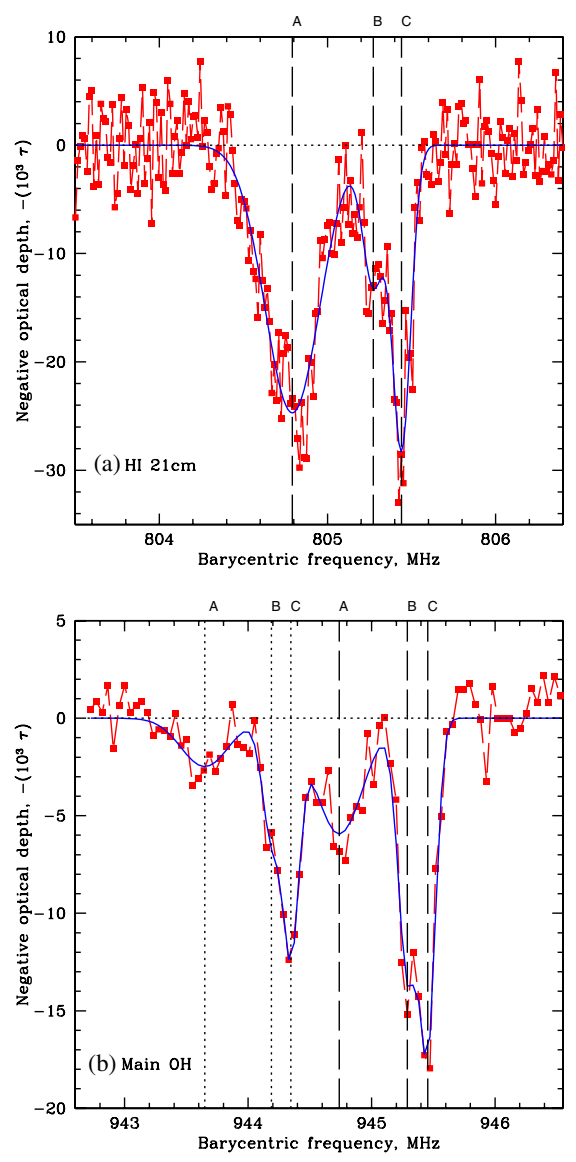

FIG. 1 (color online). GBT HI $21 \mathrm{~cm} \mathrm{[(a),} \sim 5 \mathrm{~km} / \mathrm{s}$ resolution)] and $\mathrm{OH}$ mainline [(b), $\sim 14.5 \mathrm{~km} / \mathrm{s}$ resolution] spectra toward PMN J0134-0931, with negative optical depth $\left(-10^{3} \times\right.$ $\tau)$ plotted against barycentric frequency, in MHz. The solid line shows the three-Gaussian fit to each spectrum. The vertical lines in each figure indicate the locations of the three components (marked $A, B$, and $C$ ), with the dashed and dotted lines in (b) showing the 1667 and 1665 components, respectively. tected, down to $3 \sigma$ limits of $\tau<0.07$ for the $\mathrm{HCO}^{+}$line and $\tau<0.002, \tau<0.005$ for the ground and excited state $\mathrm{H}_{2} \mathrm{CO}$ lines, respectively. This is curious as every other redshifted $\mathrm{OH}$ absorber has also been detected in $\mathrm{HCO}^{+}$ absorption [e.g., [11,15]]. While the structure of the background source is very different at the $\mathrm{HCO}^{+}$and $\mathrm{OH}$ frequencies, implying that small-scale structure in the molecular cloud could be an issue, it is still surprising that none of the $\mathrm{OH}$ components, at very different velocities from each other, show any trace of mm-wave absorption.

The HI $21 \mathrm{~cm}$ profile has three fairly clear components, two of which are blended and well separated from the third. Similarly, both the 1667 and $1665 \mathrm{MHz}$ OH lines show two clearly resolved components, with the lower redshift one somewhat asymmetric, suggesting that it is blended. While the redshifts of the two strong $21 \mathrm{~cm}$ and main $\mathrm{OH}$ components are similar, the $\mathrm{OH}$ satellite lines are blueshifted by $\sim 10 \mathrm{~km} / \mathrm{s}$ relative to the lowest redshift (highest fre-

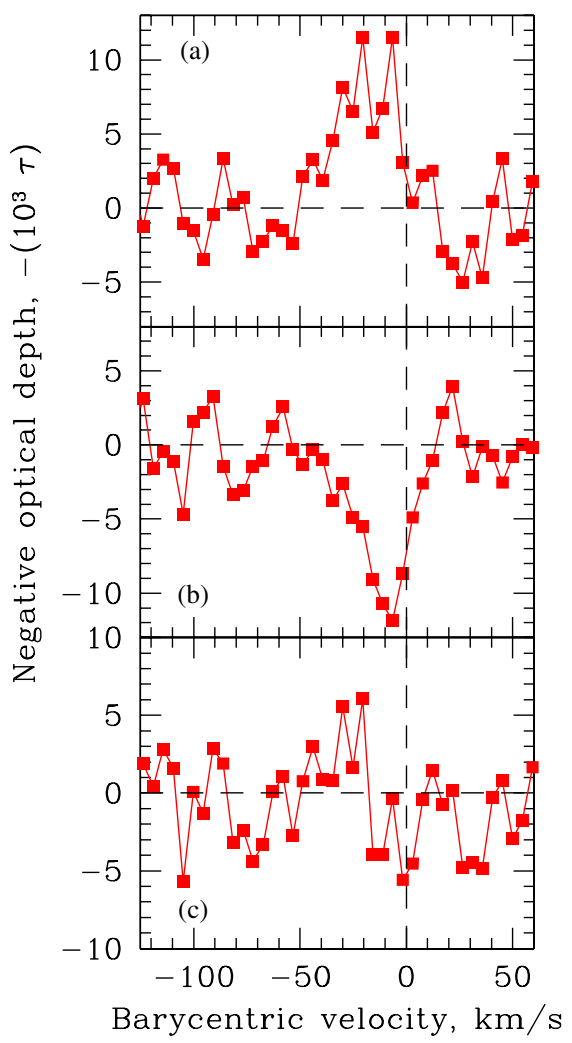

FIG. 2 (color online). $\mathrm{OH}$ satellite line spectra $(\sim 5 \mathrm{~km} / \mathrm{s}$ resolution), with negative optical depth $\left(-10^{3} \times \tau\right)$ plotted against barycentric velocity, in $\mathrm{km} / \mathrm{s}$, relative to $z=0.76355$, the redshift of component $C$. (a) $1720 \mathrm{MHz}$ transition, redshifted to $\sim 975.6 \mathrm{MHz}$; (b) $1612 \mathrm{MHz}$ transition, redshifted to $\sim 912.2 \mathrm{MHz}$; (c) sum of 1612 and $1720 \mathrm{MHz}$ spectra; this spectrum is consistent with noise. Note that the feature at $\sim-30 \mathrm{~km} / \mathrm{s}$ is not statistically significant. The satellite lines peak $\sim 10 \mathrm{~km} / \mathrm{s}$ blueward of component $C$ (the dashed vertical line), which has the lowest redshift of the three spectral components. 
quency) component in Figs. 1(a) and 1(b). This is reminiscent of the situation in the other redshifted conjugate $\mathrm{OH}$ system, PKS1413 + 135 [5]. In both cases, the sum of the satellite frequencies is different from the sum of the mainline frequencies; since the two sums have the same dependence on $\alpha, \mu$, and $g_{p}[8,9]$, the satellite and main $\mathrm{OH}$ lines must arise in different gas.

The sum of 1720 and $1612 \mathrm{MHz}$ optical depths in Fig. 2(c) is consistent with noise; the satellite lines are thus conjugate with each other. Such conjugate behavior arises due to competition between the intraladder $119 \mu$ and cross-ladder $79 \mu$ decay routes to the $\mathrm{OH}$ ground state, after the molecules have been pumped by collisions or farinfrared radiation into the higher excited states [e.g., [16] ]. The fact that the $1720 \mathrm{MHz}$ line is seen in emission and the $1612 \mathrm{MHz}$, in absorption, implies that the intraladder decay route dominates. This, and the requirement that the $119 \mu$ transition be optically thick for the $1720 \mathrm{MHz}$ line inversion [16], yields the constraint $3.5 \times 10^{15} \lesssim N_{\mathrm{OH}} \lesssim$ $3.5 \times 10^{16} \mathrm{~cm}^{-2}$ on the $\mathrm{OH}$ column density.

The $1720 \mathrm{MHz}$ line luminosity is $L_{\mathrm{OH}} \sim 3700 L_{\odot}$, making this the brightest known $1720 \mathrm{MHz}$ megamaser by more than an order of magnitude. It is also the most distant $\mathrm{OH}$ megamaser; for comparison, the furthest known $1667 \mathrm{MHz}$ megamaser is at $z \sim 0.265$ [17]. This is also the first case of conjugate $\mathrm{OH}$ satellite emission/absorption in a "normal" galaxy; all previous cases were objects containing an active galactic nucleus [e.g., Cen A; [15]], where the $\mathrm{OH}$ level populations might have been affected by nuclear activity. This is interesting as it suggests that such conjugate behavior might not be as rare as earlier expected and hence, that it might be used as a tool to probe both spatial and temporal changes in fundamental constants.

Constraining changes in fundamental constants. - A comparison between the redshifts of different spectral lines to measure changes in fundamental constants involves the assumption that the lines have no intrinsic velocity offsets from each other. This is true even for comparisons between lines of the same species as different transitions might be excited under different physical conditions and thus, in different spatial locations. This appears to be the case with the main and satellite $\mathrm{OH}$ lines here, implying that one cannot compare their redshifts to estimate changes in the different constants.

However, as in the case of PKS1413 + 135 [5,10], the conjugate nature of the satellite lines ensures that they arise in the same physical region and, crucially, that systematic velocity offsets are not an issue. The different dependences of the sum and difference of the $1720 \mathrm{MHz}$ and $1612 \mathrm{MHz}$ frequencies on $\alpha, \mu$, and $g_{p}$ then allows us to estimate changes in the quantity $G \equiv g_{p}\left[\alpha^{2} / \mu\right]^{1.85}[5,8]$. The low signal-to-noise ratio of the 1720 and 1612 spectra of Fig. 2 precludes such an estimate at the present time. However, the high redshift of the system implies that it is an excellent target for deep integrations in the satellite lines, enabling a precision measurement of changes in $\alpha, \mu$, and $g_{p}$ in the future. For example, observations with the Square Kilometer Array, a next generation radio telescope, should be able to detect fractional changes $\Delta G / G \sim 5 \times 10^{-7}$ in this system, implying a sensitivity of $\Delta \alpha / \alpha \sim 1.4 \times 10^{-7}$. This is similar to the sensitivity of the Oklo measurement but with fewer assumptions and out to a far larger lookback time of $\sim 6.7 \mathrm{G} \mathrm{yr}$. In addition, this sensitivity would be obtained from a single system, unlike the many-multiplet method, which requires a large number of absorbers to average out systematic effects. A comparison between the results from the conjugate systems in PKS1413+ 135 and PMN J0134-0931 will thus allow one to probe true spatiotemporal changes in the above constants (instead of merely averaging over spatial effects), especially since the two sources are very widely separated on the sky.

Comparisons between the $\mathrm{HI} 21 \mathrm{~cm}$ and main $\mathrm{OH}$ lines suffer from the drawback of possible systematic velocity offsets between the two species. However, a tight correlation has been found between the velocities of $\mathrm{HCO}^{+}$and nearest HI $21 \mathrm{~cm}$ absorption in the Galaxy, with a dispersion of only $\sim 1.2 \mathrm{~km} / \mathrm{s}$ [18]. Further, the velocities of Galactic $\mathrm{OH}$ and $\mathrm{HCO}^{+}$absorption have been found to be remarkably similar [[19]; see Figs. 4 and 5 of [19] ]. OH and $\mathrm{HI} 21 \mathrm{~cm}$ velocities should thus also be well correlated and, in fact, the dispersion in this correlation is likely to be less than that between the $\mathrm{HCO}^{+}$and $\mathrm{HI} 21$ velocities, as the spatial structure of the background source is quite similar at the nearby $\mathrm{OH} 18 \mathrm{~cm}$ and $\mathrm{HI} 21 \mathrm{~cm}$ frequencies. It should thus be possible to use a comparison between main $\mathrm{OH}$ and $\mathrm{HI} 21 \mathrm{~cm}$ absorption from a statistically large number of redshifted systems as an independent probe of any evolution in $\alpha, \mu$, and $g_{p}$ [8]. We next apply this technique to the absorbers toward PMN J0134-0931 and B0218 + 357, albeit using a more conservative dispersion of $3 \mathrm{~km} / \mathrm{s}$ between $\mathrm{OH}$ and $\mathrm{HI}$ intrinsic velocities, characterizing internal motions within a molecular cloud.

The HI $21 \mathrm{~cm}$ profile of Fig. 1(a) has the highest signalto-noise ratio of our spectra (at the same resampled resolution of $\sim 1 \mathrm{~km} / \mathrm{s}$ ) and has three clear components. We hence used a three-Gaussian template to locate the peak redshifts of the different $21 \mathrm{~cm}$ absorption components, with the amplitudes, positions, and widths of the Gaussians all left as free parameters. A similar three-Gaussian fit was then carried out to the smoothed and resampled 1665 and $1667 \mathrm{MHz}$ spectra, with the difference that the velocity widths here were fixed to those obtained from the $21 \mathrm{~cm}$ fit and only the amplitudes and positions of the individual components left as free parameters. The 1665 and $1667 \mathrm{MHz}$ fits were carried out simultaneously, to account for the possibility of blending between the components. We note that the original spectral resolution was $\sim 1 \mathrm{~km} / \mathrm{s}$ in all cases and no additional components were seen in any of the spectra. The possibility that strong narrow compo- 
nents might be blended in the lower resolution spectra of Figs. 1(a) and 1(b) can thus be ruled out.

Figures 1(a) and 1(b) show the fitted Gaussians as solid lines, overlaid on the $\mathrm{HI}$ and $\mathrm{OH}$ spectra. The three fitted $21 \mathrm{~cm}$ components have peak redshifts $z_{\mathrm{HI}-\mathrm{A}}=$ $0.764938 \pm 0.000015, \quad z_{\mathrm{HI}-\mathrm{B}}=0.763881 \pm 0.000033$, and $z_{\mathrm{HI}-\mathrm{C}}=0.763515 \pm 0.000014$, while the sums of the 1665 and $1667 \mathrm{MHz}$ frequencies have peak redshifts $z_{\mathrm{OH}-\mathrm{A}}=0.764871 \pm 0.000042, \quad z_{\mathrm{OH}-\mathrm{B}}=0.763852 \pm$ 0.000019 , and $z_{\mathrm{OH}-\mathrm{C}}=0.763550 \pm 0.000010$. Comparing these redshifts, component by component, we obtain $[\Delta F / F]_{\mathrm{A}}=(-3.7 \pm 2.8) \times 10^{-5}, \quad[\Delta F / F]_{\mathrm{B}}=$ $(-1.6 \pm 2.1) \times 10^{-5}, \quad$ and $\quad[\Delta F / F]_{\mathrm{C}}=(2.0 \pm 1.0) \pm$ $10^{-5}$, where $F \equiv g_{p}\left[\alpha^{2} / \mu\right]^{1.57}$ and only statistical errors have been included. A weighted average of these values gives $[\Delta F / F]=(0.86 \pm 0.86) \times 10^{-5}$. Note that a comparison using an entirely unconstrained six-Gaussian fit to the $\mathrm{OH}$ spectrum gives the weighted average $[\Delta F / F]=$ $(2.25 \pm 0.84) \times 10^{-5}$. Similarly, the HI $21 \mathrm{~cm}$ and main $\mathrm{OH}$ redshifts in the $z \sim 0.685$ lens toward $\mathrm{B} 0218+$ 357 are $z_{\mathrm{HI}}=0.684676 \pm 0.000005[20]$ and $z_{\mathrm{OH}}=$ $0.684682 \pm 0.0000056 \quad[8]$, giving $[\Delta F / F]_{\mathrm{D}}=$ $(3.5 \pm 4.0) \times 10^{-6}$. All the above values are consistent with the null hypothesis of no evolution in the different constants. Combining results from the two absorbers (using the constrained fit and a weighted average), we obtain $[\Delta F / F]=\left(0.44 \pm 0.36^{\text {stat }} \pm 1^{\text {syst }}\right) \times 10^{-5}$, over $0<z \lesssim$ 0.7 , where the second error is due to velocity offsets between $\mathrm{HI}$ and $\mathrm{OH}$ lines, assuming a velocity dispersion of $3 \mathrm{~km} / \mathrm{s}$. Of course, four measurements are far too few for a reliable estimate of this error. However, the fact that the $\mathrm{OH}$ and $\mathrm{HI}$ redshifts are in reasonable agreement within the measurement errors in all four cases (two of which have errors $\leqslant 1 \times 10^{-5}$ ) suggests that systematic velocity offsets do not dominate the accuracy of the measurement.

The strong dependence of $F$ on $\alpha$ and $\mu\left(F \propto \alpha^{3.14}\right.$ and $F \propto \mu^{-1.57}$ ) implies a $2 \sigma$ sensitivity of $[\Delta \alpha / \alpha]<6.7 \times$ $10^{-6}$ or $[\Delta \mu / \mu]<1.4 \times 10^{-5}$ to fractional changes in $\alpha$ and $\mu$ from $z \sim 0.7$ (i.e., a lookback time of $\sim 6.5$ Gyrs) to today, where we have added the errors in quadrature (these sensitivities are not independent, as we measure $[\Delta F / F]$ ). Assuming linear evolution, these correspond to $2 \sigma$ sensitivities of $(1 / \alpha)[\Delta \alpha / \Delta t]<1.1 \times 10^{-15} \mathrm{yr}^{-1}$ or $(1 / \mu) \times$ $[\Delta \mu / \Delta t]<2.1 \times 10^{-15} \mathrm{yr}^{-1}$, among the best at present to changes in $\mu$. For comparison, [7] obtains $[\Delta \mu / \mu]<$ $1.48 \times 10^{-5}$ for $0<z \lesssim 2.75$ while [6] obtains $[\Delta \mu / \mu] \lesssim 2 \times 10^{-5}$ for $0<z<2.0$, at $2 \sigma$ level, using redshifted optical lines. The present radio analysis is not affected by two important sources of systematic error in the optical regime [wavelength uncertainties and relative isotopic abundances; e.g., [3] ], although, of course, the optical observations probe a larger redshift range. The primary source of the error in our technique is likely to lie in the velocity dispersion between $\mathrm{OH}$ and $\mathrm{HI}$ lines and perhaps in blending between weak narrow spectral components. We do not feel that these dominate the present results; deeper observations in the $\mathrm{OH}$ and $\mathrm{HI}$ lines should help quantify their effects.

While the size of the radio sample is still small, surveys are being carried out that will significantly increase the number of known redshifted $\mathrm{OH}, \mathrm{HI}$, and $\mathrm{HCO}^{+}$absorbers. Comparisons between radio lines are thus likely to provide an important independent constraint on changes in fundamental constants in the future.

We thank Bob Garwood and Jim Braatz for help with the data analysis. The National Radio Astronomy Observatory is operated by Associated Universities, Inc., under cooperative agreement with the National Science Foundation. The Australia Telescope Compact Array is part of the Australia Telescope, funded by the Commonwealth of Australia for operation as a National Facility managed by CSIRO.

*Electronic address: nkanekar@aoc.nrao.edu

[1] E. Peik, B. Lipphardt, H. Schnatz, T. Schneider, C. Tamm, and S. G. Karshenboim, Phys. Rev. Lett. 93, 170801 (2004).

[2] T. Damour and F. J. Dyson, Nucl. Phys. B480, 37 (1996).

[3] M. T. Murphy, J. K. Webb, and V. V. Flambaum, Mon. Not. R. Astron. Soc. 345, 609 (2003).

[4] R. Srianand, H. Chand, P. Petitjean, and B. Aracil, Phys. Rev. Lett. 92, 121302 (2004).

[5] N. Kanekar, J. N. Chengalur, and T. Ghosh, Phys. Rev. Lett. 93, 051302 (2004).

[6] P. Tzanavaris, J. K. Webb, M. T. Murphy, V. V. Flambaum, and S. J. Curran, Phys. Rev. Lett. 95, 041301 (2005).

[7] A. Ivanchik et al., Astron. Astrophys. 440, 45 (2005).

[8] J. N. Chengalur and N. Kanekar, Phys. Rev. Lett. 91, 241302 (2003).

[9] J. Darling, Phys. Rev. Lett. 91, 011301 (2003).

[10] J. Darling, Astrophys. J. 612, 58 (2004).

[11] N. Kanekar and J. N. Chengalur, Astron. Astrophys. 381, L73 (2002).

[12] N. Kanekar, J. N. Chengalur, A. G. de Bruyn, and D. Narasimha, Mon. Not. R. Astron. Soc. 345, L7 (2003).

[13] Throughout this Letter, we use an LCDM cosmology, with $\Omega_{m}=0.3, \Omega_{\Lambda}=0.7$, and $H_{0}=70 \mathrm{~km} / \mathrm{s} \mathrm{Mpc}^{-1}$.

[14] N. Kanekar and F.H. Briggs, Astron. Astrophys. 412, L29 (2003).

[15] T. Wiklind and F. Combes, Astrophys. J. 500, 129 (1998).

[16] H. J. van Langevelde, E. F. van Dishoek, M. N. Sevenster, and F. P. Israel, Astrophys. J. 448, L123 (1995).

[17] W. A. Baan, J. Rhoads, K. Fisher, D. R. Altschuler, and A. Haschik, Astrophys. J. 396, L99 (1992).

[18] M. J. Drinkwater, J. K. Webb, J.D. Barrow, and V. V. Flambaum, Mon. Not. R. Astron. Soc. 295, 457 (1998).

[19] H. Liszt and R. Lucas, Astron. Astrophys. 355, 333 (2000); H. Liszt (private communication).

[20] C. L. Carilli et al., Phys. Rev. Lett. 85, 5511 (2000). 